\title{
Coronary Intervention Planning Using Hybrid 3D Reconstruction
}

\author{
O. Wink ${ }^{12}$, R. Kemkers ${ }^{1}$, S.J. Chen ${ }^{2}$, and J.D. Carroll ${ }^{2}$ \\ 1 Cardiovascular X-ray, Philips Medical Systems North America, \\ Bothell, Washington, 98041 USA \\ \{onno.wink, richard.kemkers\}@philips.com \\ 2 Division of Cardiology, Department of Medicine, \\ University of Colorado Health Sciences Center, Denver, Colorado, 80262 USA \\ $\{$ james.chen, john. carroll\}@uchsc. edu
}

\begin{abstract}
A new method is presented to assist the clinician in planning a interventional procedure while the patient is already on the catheterization table. Based on several ECG-selected projections from a rotational $\mathrm{X}$-ray acquisition, both a volumetric cone-beam reconstruction of the coronary tree as well as a three-dimensional surface model of the vessel segment of interest are generated. The proposed method provides the clinician with the length and diameters of the vessel segment of interest as well as with an 'optimal working view'. In this view, the gantry is positioned such that the vessel segment of interest is the least foreshortened and vessel overlap is minimized during the entire heart cycle. Examples on a phantom object and on patient data demonstrate the accuracy and feasibility of the approach.
\end{abstract}

\section{Introduction}

Coronary artery disease remains the major cause of morbidity and mortality in the United States. Over the past years, there has been a marked increase in the number of catheterizations procedures performed. The number of catheterizations is expected to furher increase due to recent advances in stent technology (e.g. eluting stent), imaging capabilities and an aging of the polulation. Coronary catheterizations are performed using X-ray angiography. Choosing the correct stent dimensions is often difficult using the traditional 2D projection images due to vessel foreshortening and overlap. Based on the specific anatomy of the coronary branch and skill of the clinician, several angiographic images from different viewing angles are acquired in order to derive the length and diameter of the stent to be used for subsequent intervention. Once the clinician decides upon the stent dimensions, a 'working view' is chosen for the actual interventional procedure. In this view, it is expected that the vessel segment of interest is the least foreshortened and not blocked from sight. This 'trial-and-error' method of selection of appropriate viewing angles potentially exposes the patient to large amounts of contrast medium (dye) and radiation, even before the actual treatment of the patient begins. 
In order to avoid the 'trial-and-error' approach, several researchers have proposed various methods to construct a three-dimensional surface model of the coronaries using two or three acquisitions from different viewing angles (e.g. [1, 2, 3, 4,5]), generally needing considerable user interaction. As a result, the clinician has the ability to view the entire three-dimensional coronary tree from any angle and to choose the 'optimal working view' without the use of extra radiation or dye.

In the last few years, rotational angiography (RA) has proven to be a very accurate and effective diagnostic tool in the treatment of cerebral vessel malformations. In this approach, the $\mathrm{C}$-arm rotates rapidly around the patient while several X-ray projections are acquired. The reconstructed vessels can be viewed from different viewing angles, while only one contrast injection is given. It has been demonstrated that the use of rotational angiography for coronary vessel acquisitions yields better stenosis severity estimations and reveals lesions that were missed by only applying the traditional acquisitions [6]. Due to the high reproducibility of the rotational acquisitions, the fast rotation speed, and the nature of the cerebral vessels, the projections can be used for volumetric reconstruction providing very high detail and accuracy [7, and is completely automated.

However, straightforward volumetric reconstruction of coronary arteries has several difficulties due to the beating of the heart and respiratory motion, yielding a rough representation of the coronaries (see also left frame of Fig. 2). Although recent developments with alternative reconstruction schemes are very promising [8], an accurate reconstruction that can directly be used for lesion sizing in human subjects is not yet available. Even if a suitable volumetric reconstruction could be performed, additional user interaction is needed to arrive at the correct stent dimensions.

In this paper, a method is proposed that combines the complementary features of volume -and surface based reconstruction techniques. The rotational acquisition is used to minimize contrast medium and X-ray exposure. The projections that correspond to the same phase of the cardiac cycle are used to create an accurate surface-based model of the coronary segment of interest and to create an overview of the main coronary vessels. The presented method is unique in several ways. First, the creation of a surface model of the coronaries based on projections from a rotational acquisition has not been demonstrated before. Second, the method allows a model to be built based on every projection that captured the heart at the same phase. Third, the combination with the automatic volumetric reconstruction can be used for visualization of the main vessels of the coronary tree and to determine the 'optimal working view' without vessel overlap and without the need to manually create a surface model of the entire coronary tree. Fourth, to our knowledge, a unique study is presented about the volumetric reconstruction of the main coronary arteries of actual patients with the use of a C-arm system.

In Section 2 the method is described in more detail and the concept of the 'Optimal View Map' is explained. In Section 3 the method is applied to a static 


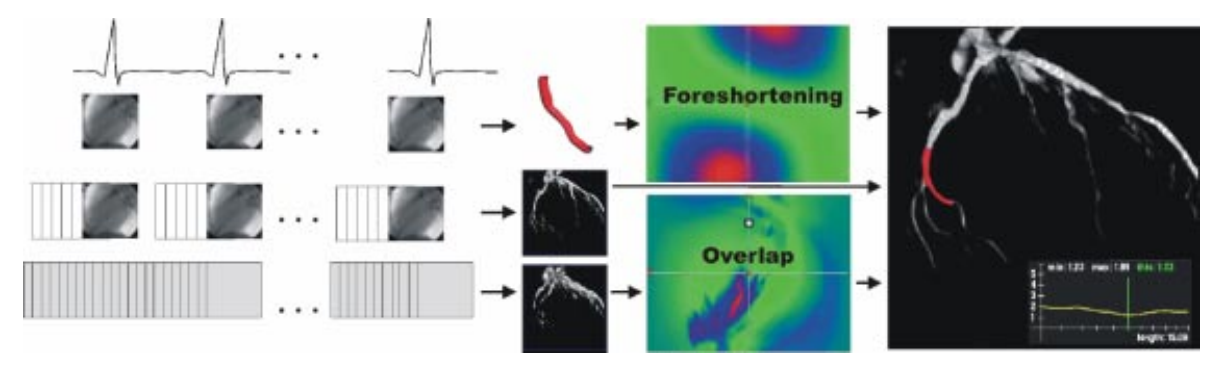

Fig. 1. Flow of the method resulting in the dimensions of a coronary segment and an optimal viewing angle to perform the actual intervention.
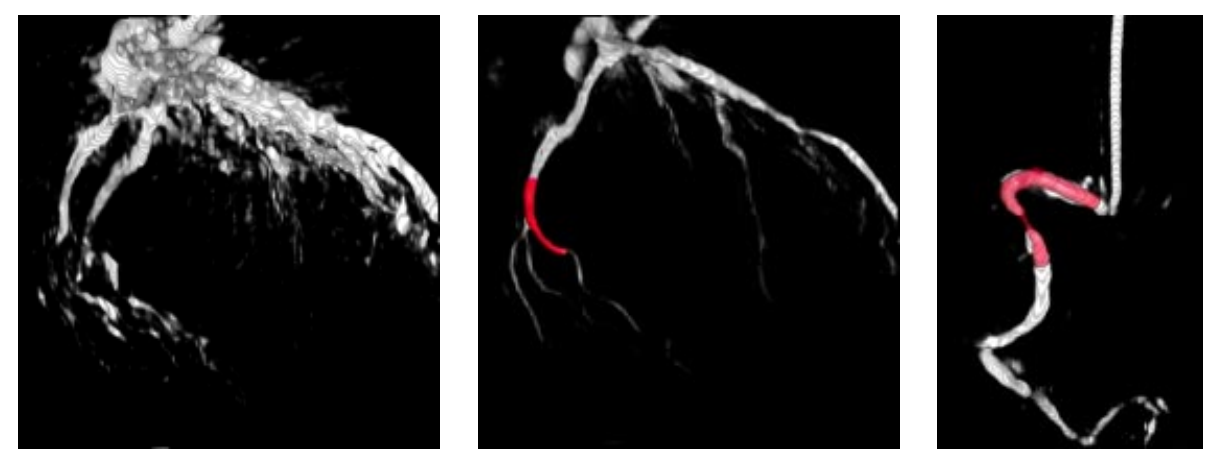

Fig. 2. Reconstruction using all projections (left). Hybrid visualization of a surface model and volumetric reconstruction based on ECG based selection of projections (middle and right).

phantom and a catheter with radiopaque markers in a patient to asses its accuracy. The discussion and conclusions are given in Section 4 .

\section{Description of the Method}

In Fig. 1 the different phases of the method are shown. After the X-ray projections are acquired using a rotational C-arm system (Philips Integris Allura 12 inch monoplane), the images are sent to a workstation (Silicon Graphics Octane). Based on the ECG signal, two projections are chosen that correspond to the same phase of the cardiac cycle to create the central vessel axis followed by the computation of the foreshortening map. Other projections captured at the same phase can be used to refine the surface model of the segment of interest. The projections that are approximately of the same phase are used to visualize the coronary tree, while all the acquired projections are used to compute the overlap map. 


\subsection{Acquisition}

The C-arm is placed near the head-position of the table in order to perform a calibrated propeller acquisition from $120 \mathrm{RAO}$ (right side of patient) to $120 \mathrm{LAO}$ (left side of patient). The C-arm rotates at 55 degrees per second and acquires images at 30 frames per second during 4 seconds while the patient is asked to hold his/her breath. A total of 8 to 12 cc's of contrast medium (Omnipaque 350 ) is injected during the acquisition and started 1 second before the gantry starts rotating. Since this acquisition has been calibrated in an earlier stage (see [7), the individual projections can be corrected for the earth magnetic field and pincushion distortion and transformed to a common world coordinate system.

\subsection{Surface-Based Modeling}

Based on the ECG signal, the projections that correspond to the same phase of the cardiac cycle are chosen to model the segment of the artery of interest. To construct the central axis, those projections are chosen where the segment of interest is clearly visible and the angle between the two projections is around 90 degrees. Since on average five to six heartbeats are captured during the run, the user generally can choose the corresponding projections with a clear view of the lesion. The central axis of the arterial segment is manually identified by adding point pairs. A point in projection A yields an epipolar line in projection $\mathrm{B}$, which is the ray coming from projection A towards the X-ray source. Once a point is set in one projection, the user has to define the corresponding point along the epipolar line in the other projection to construct the central axis of the model. By using this 'epipolar constraint', it is guaranteed that the corresponding epipolar lines do intersect and define a 3D axis point. Once the axis of the lesion is created, the user has the opportunity to delineate the borders of the lumen in every projection that corresponds to the same phase of the heart in order to create and refine a surface model of the lumen. The average time to create a model for a segment of interest once two corresponding projections are found is less than a minute on a SGI Octane.

\section{$2.3 \quad$ Volumetric Reconstruction}

An adapted version of the Feldkamp backprojection [9] algorithm is applied for the volumetric reconstruction. The projections are weighted according to the speed of the C-arm, where the projections acquired at a constant rotation speed have a higher weight than those acquired during startup and slowing down of the $\mathrm{C}$-arm. If all available projections are used, the reconstruction contains information about several phases of the heart simultaneously, and is often difficult to interpret (left frame of Fig. (2). If only those projections are used that correspond to the same time point in the cardiac cycle used to build the surface model, the reconstructed volume is better suited for simultaneous visualization and inspection of the artery (middle and right frame of Fig. (2)). 


\subsection{Optimal View Determination}

The view that is chosen to perform the intervention is based on a combination of the amount of foreshortening of the segment of interest and the overlap of other structures [2]. The idea behind using a map is that only those gantry angles are computed that can actually be achieved. In general, the cranial (toward the head of the patient) and caudal (toward the feet of the patient) angulation should not exceed 45 degrees from the anterior-posterior (AP) plane since the image intensifier is very likely to hit the patient otherwise. The RAO and LAO thresholds can be set according to the capabilities of the imaging system and are currently set to a maximum of 60 degrees from the AP plane to avoid collision of the image intensifier with the operating table or the patient. The different values within the predefined range of angles are visualized using an interactive color coded map. In the examples used in this paper, a green color in the maps corresponds to the best viewing angle while the red color corresponds to the worst viewing angle. The left of the map corresponds to a RAO position (right with LAO) while the top of the map corresponds to a cranial position (bottom with caudal). The user has the opportunity to inspect the different viewing angles in the map by clicking at a position in the map and to toggle between the different maps. The amount of foreshortening and overlap are given in the lower right hand side of the viewport.

Foreshortening Map. The foreshortening map is computed by comparing the length of the modeled segment to the projected length of this segment as if it were viewed from a typical viewpoint as defined by the range of angles in the map [2].

Overlap Map. Although the volumetric reconstruction of all the acquired projections yields a rough representation of the coronaries, it does provide information where the vessels and other objects (e.g. the spine, ribs and pacemaker or ECG-leads) are located during all the phases of the heart. The overlap map is computed by taking the integral of all the grey values from the reconstructed volume along the rays that intersect the modeled segment. This is very efficiently implemented in OpenGL using space-leaping techniques and the Stencil Buffer [10] in combination with the special purpose graphics hardware of the SGI Octane.

Optimal View Map. The optimal view map can be constructed by taking a weighted sum of normalized values of the foreshortening map and overlap map (see Fig. 3). For a practical implementation and efficient computation, the vessel overlap is generally only estimated for those gantry angles where the vessel foreshortening has been minimized in order to obtain the resultant optimal working view. 

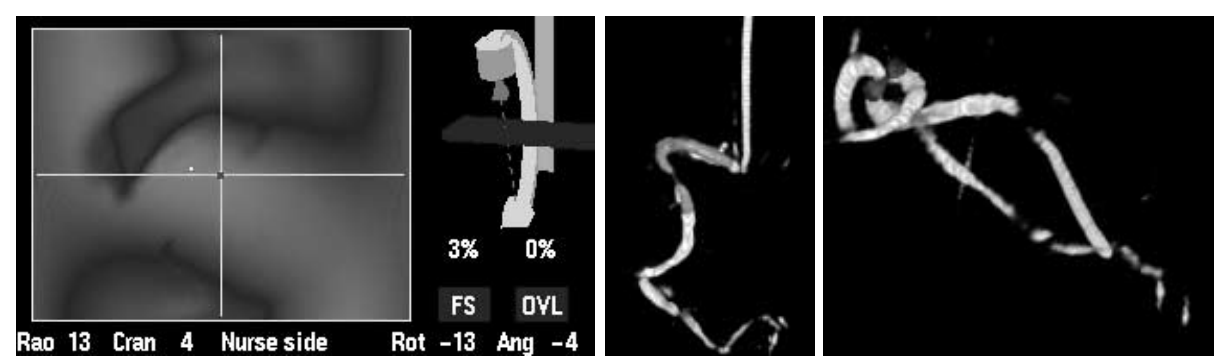

Fig. 3. Example of the optimal view map and the corresponding gantry configuration. The middle and right frame correspond to the best and worst viewing angle as determined by the the optimal view map.

\section{Evaluation}

Although the method has been applied to over 40 patients, the evaluation focuses on the accuracy and the feasibility of the proposed approach. A phantom with known dimensions is employed for accuracy assessment on length and diameter and the overlap map. Furthermore, the accuracy of the modeling approach is investigated using a marked catheter placed in the left ventricle of the heart.

\subsection{Phantom}

The employed phantom is a perspex cube of $3 \times 3 \times 3 \mathrm{~cm}$, containing three aluminum rods with a diameter of $2 \mathrm{~mm}$, oriented in the three main directions and 3 spheres with a diameter of $3 \mathrm{~mm}$ as shown in figure 4 . The 13-th and 63-th projection are chosen from the rotational acquisition to model the rod that is vertically positioned. To quantify the accuracy, four borders are carefully drawn to define the associated diameter of the rods. All the projections are used to reconstruct the volume for both visualization and computation of the overlap map. The projection corresponding to the worst viewing angle is shown in the right frame of figure 4 From the hybrid visualization in the right frame of figure 4 it can be derived that the generated model is very similar to the reconstructed volume. Four different propeller acquisitions are performed with the image-intensifier set to $12,9,7$ and 5 inch, respectively. The average diameter of the modeled rod is $1.84 \mathrm{~mm}$, while the average length of the rod is $30.02 \mathrm{~mm}$. The underestimation of the diameter of the rod can be explained by the reduced contrast between the material of the rod and its surrounding perspex. This is not the case in the determination of the rod's length which is therefore much more accurate.

\subsection{Marked Catheter}

A marked catheter (Cook Royal Flush II pigtail) is placed in the left ventricle of the heart of two patients. The catheter has two radio-opaque markers at the tip that are $22 \mathrm{~mm}$ apart (see left and middle frame of Fig. 51). Based on 

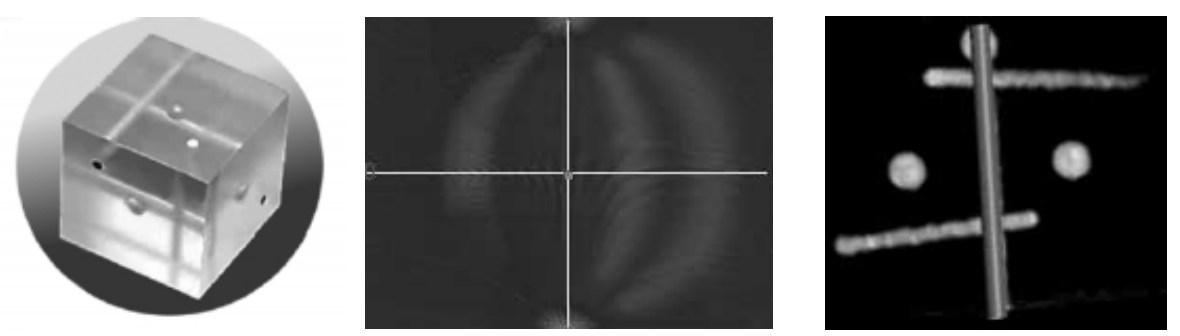

Fig. 4. The perspex phantom (left frame). The overlap map (middle frame), clearly displaying the 'orbits' of the spheres where they are overlapping with the rod of interest. Hybrid visualization of the modeled (vertical) rod and reconstructed phantom having the most overlap (right frame).
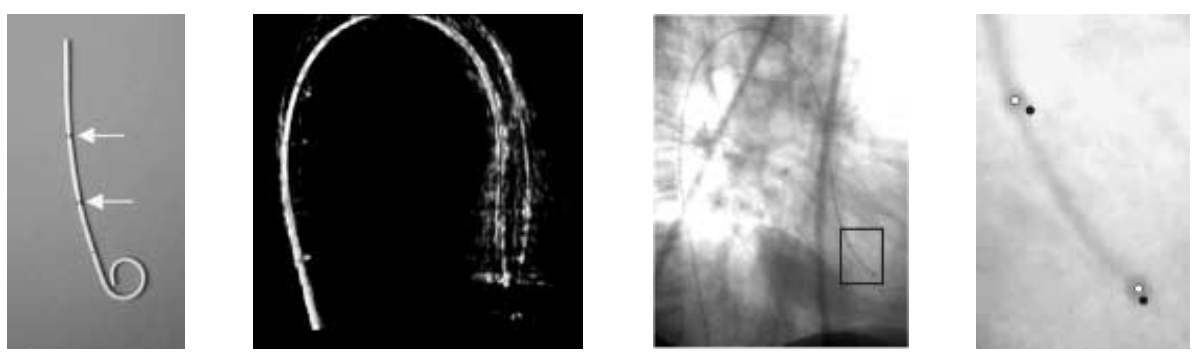

Fig. 5. The catheter with the radio-opaque markers (leftmost frame). The volumetric reconstruction displaying the movement of the catheter tip (middle left frame). A projection displaying the markers while positioned in the left ventricle of a patient (middle right frame). The determination of the accuracy of the projected 3D position of the markers (black dots) compared to the manually determined position of the markers (white dots in rightmost frame).

two projections $\mathrm{A}$ and $\mathrm{B}$ from the rotational acquisitions using an II-size of 12 inch, the $3 \mathrm{D}$ location of the markers is determined. The resulting $3 \mathrm{D}$ position is projected on the third projection $(\mathrm{C})$ that corresponds to the same phase of the heart as projections $\mathrm{A}$ and $\mathrm{B}$. The average difference between the projected $3 \mathrm{D}$ points and the position of the corresponding markers in projection $\mathrm{C}$ for both patients is 3.52 pixels.

\section{Discussion and Conclusions}

A hybrid reconstruction scheme is proposed that provides the clinician with the three-dimensional quantitative measurement of an arterial segment and an optimal working view throughout the cardiac cycle. This information can be provided in less than a minute and used to plan the intervention while the patient is still on the catheterization table. The accuracy of the method is validated using a static phantom and a marked catheter placed in a beating heart during a rotational acquisition. The study found the modeling approach to be accurate if the 
segment of interest is clearly visible in the different projections that correspond to the same time point in the heart cycle.

\section{Acknowledgments}

We would like to acknowledge Rolf Suurmond, Paul Desmedt and Wiet Lie from Philips X-ray pre-development in Best, The Netherlands, for their active support in creating the prototype environment. Babak Movassaghi and Reiner Koppe from Philips Research Laboratories in Hamburg are acknowledged for their help on calibration issues. The many anonymous patients are respectfully acknowledged for consenting in the use of their image data. Franklin Schuling is acknowledged for establishing the cooperation between Philips Research and the Cardiac Cath Lab of the University of Colorado Health Sciences Center.

\section{References}

1. S. J. Chen and J. D. Carroll, "3-D reconstruction of coronary arterial tree to optimize angiographic visualization," IEEE Transactions on Medical Imaging, vol. 9, no. 4, pp. 318-336, April 2000.

2. S. -Y. J. Chen and J. D. Carroll, "Computer assisted coronary intervention by use of on-line 3D reconstruction and optimal view strategy," in Proc. Medical Image Computing and Computer-Assisted Intervention, 1998, pp. 377-385.

3. A. Wahle, E. Wellnhofer, I. Mugaragu, H. U. Sauer, H. Oswald, and E. Fleck, "Assesment of diffuse coronary artery disease by quantitative analysis of coronary morphology based upon 3-D reconstruction from biplane angiograms," IEEE Transactions on Medical Imaging, vol. 14, no. 2, pp. 230-241, June 1995.

4. A. C. M. Dumay, J. H. C. Reiber, and J. J. Gerbrands, "Determination of optimal angiographic viewing angles: Basic principles and evaluation study," IEEE Transactions on Medical Imaging, vol. 12, no. 1, pp. 13-24, 1994.

5. U. Solzbach, U. Oser, M. Romback, H. Wollschläger, and H. Just, "Optimum angiographic visualization of coronary segments using computer-aided 3D reconstruction from biplane views," Computers and Biomedical Research, vol. 27, pp. 178-198, 1994.

6. G. Tommasini, A. Camerini, A. Gatti, G. Derchi, A. Bruzzone, and C. Vecchio, "Panoramic coronary angiography," JACC, vol. 31, no. 4, pp. 871-877, March 1998.

7. R. Koppe, E. Klotz, J. op de Beek, and H. Aerts, "Digital stereotaxy/stereotactic procedures with C-arm based rotational-angiography (RA)," in Computer Assisted Radiology, H. U. Lemke et al., Ed. 1996, pp. 17-22, Elsevier Publishers, Amsterdam.

8. V. Rasche, A. Buecker, M. Grass, R. Koppe, J. op de Beek, R. Bertrams, R. Suurmond, H. Kuehl, and R.W. Guenther, "ECG-gated 3D-rotational coronary angiography," in Proc. Computer Assisted Radiology and Surgery, H. U. Lemke, Ed., 2002, accepted for publication.

9. L. A. Feldkamp, L. C. Davis, and J. W. Kress, "Practical cone-beam algorithms," Journal of the Opt. Society of America, vol. A, no. 6, pp. 612-619, 1984.

10. M. Woo, J. Neider, T. David, and D. Shreiner, OpenGL Programming Guide, Version 1.2, Addison Wesley, Reading, Mass, 1999. 